\title{
Seria o Ancylostoma caninum um dos agentes da neurorretinite sub-aguda difusa unilateral (D.U.S.N.) no Brasil?
}

Would Ancylostoma caninum be one of the agents of diffuse unilateral subacute
neuroretinitis (D.U.S.N in Brazil?

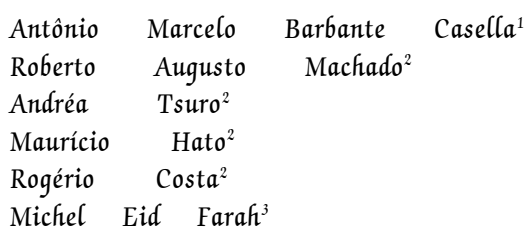

\section{RESUMO}

Os autores apresentam um caso de neurorretinite subaguda difusa unilateral(D.U.S.N) confirmada com a identificação da larva em uma criança de 6 anos associada a quadro de larva migrans cutânea, bem como a análise de outros sete casos de D.U.S.N. e sua associação sorológica à toxocaríase e antecedentes de larva migrans cutânea. Este é o primeiro caso descrito na literatura de D.U.S.N. concomitante a larva migrans cutânea na fase ativa.

Descritores: Neurite óptica/etiologia; Retinite/etiologia; Infecções oculares parasitárias/ etiologia; Larva migrans/parasitologia; Ancylostoma/sorologia

\section{INTRODUÇÃO}

A neurorretinite difusa subaguda unilateral (D.U.S.N.) é uma doença causada por um único nematódio móvel que se localiza no espaço subretiniano, podendo permanecer neste local por quatro anos ou mais que afeta adultos jovens, saudáveis, preferencialmente do sexo masculino, e caracteristicamente unilateral que leva à cegueira nas suas fases avançadas descrito por Gass et al. ${ }^{(1)}$. O quadro clínico, nas suas fases iniciais, pode mostrar perda aguda da acuidade visual associada a vitreíte, papiledema e lesões esbranquiçadas ao nível da retina externa, geralmente as lesões são agrupadas sendo esta a região onde pode ser encontrado o nematódio; nas suas fases tardias a atrofia ótica, estreitamento arteriolar, alterações degenerativas do epitélio pigmentário da retina e a baixa acuidade visual são característicos $^{(1,2)}$. O diagnóstico diferencial deve ser feito com várias patologias retinianas como a epiteliopatia placóide, coroidite multifocal por toxoplasmose, coroidite serpiginosa, doença de Behçet, pseudo síndrome da histoplasmose presumida, sarcoidose, abscesso retiniano entre outras na sua fase inicial e a retinose pigmentar unilateral, coriorretinopatia traumática, síndrome da histoplasmose presumida, atrofia retiniana pós oclusão da artéria central da retina na sua fase tardia ${ }^{(1,2)}$.

O diagnóstico precoce associado ao tratamento por meio da destruição da larva com fotocoagulação são de suma importância para a recuperação visual do paciente, o uso de anti-helmínticos permanece controverso. Casella et al., em 1998, utilizaram ivermectina e tiabendazol em casos confirmados com a identificação da larva sem sucesso sendo necessária a fotocoagulação da larva ${ }^{(3)}$. Existe na literatura uma controvérsia sobre seu agente causal. Segundo Gass e Braunstein ${ }^{(2)}$ a etiologia seria devido a nematóides de dois tamanhos de larvas o primeiro medindo entre 400 e 1000 micra sendo o responsável pelos casos de D.U.S.N. na América do Sul, Caribe e sul dos
${ }^{1}$ Professor Adjunto Doutor do Setor de Oftalmologia, Universidade Estadual de Londrina.

${ }^{2}$ Ex-residente do Setor de Oftalmologia, Universidade Estadual de Londrina.

${ }^{3}$ Professor Livre Docente do Departamento de Oftalmologia da UNIFESP-EPM.

Endereço para correspondência: Av. Bandeirantes, 500 - Sala 110 - Londrina (PR) CEP 86010-010. E-mail: mcasella@sercomtel.com.br 
Estados Unidos e o segundo medindo entre 1500 e 2000 micra sendo o responsável pelos casos no noroeste e meio oeste dos Estados Unidos, sugere ainda que o menor verme poderia ser o Ancylostoma caninum. O Baylisascaris, um nematódio de tamanho entre 300 e 2000 micra que infesta guaxinins e gambás, já foi investigado em casos de D.U.S.N. em huma$\operatorname{nos}^{(1)}$ e em modelos experimentais animais ${ }^{(4)}$, pelo seu tamanho e morfologia seria o agente nos casos em que a larva é a maior. Há ainda a Alaria mesocercaria ${ }^{(5)}$, um trematódio que foi isolado do vítreo de um paciente com quadro de D.U.S.N. e em análise de fotos do fundo de olho de outro caso, já foi aventada como etiologia da neurorretinite. No Brasil a primeira descrição da doença foi realizada por Oliveira e Oréfice ${ }^{(6)} \mathrm{em}$ sete casos de DUSN presumível. A identificação da larva foi feita por Souza e Cunha, em 1992(7), e em 1995 Souza e Nakashi$\mathrm{ma}^{(8)}$ retiraram o nematóide subretiniano sendo o mesmo compatível com o estágio terciário da larva do Toxocara canis pelo seu tamanho e morfologia. Recentemente foi relatado um caso de doença bilateral ${ }^{(9)}$.

Os agentes causadores da larva migrans cutânea são de maneira geral Ancilostomideos, incluindo o Ancilostoma caninum, o Ancilostoma braziliense e outros ancilostomídeos ${ }^{(10)}$. Os autores apresentam um caso de DUSN associado a larva migrans cutânea ativa sendo o primeiro relatado na literatura bem como o levantamento retrospectivo de outros 7 casos.

MÉTODOS

Relata-se um caso de uma criança de seis anos de idade portadora de DUSN associado a larva migrans cutânea ativa, com história de repetidas infestações de pele pela larva migrans, além de uma revisão de outros sete casos conduzidos por um dos autores com associação à sorologia para toxocaríase e história pregressa de larva migrans cutânea.

\section{Relato do caso}

A. C. O., masculino, 6 anos, estudante, natural de Paranavaí $P R$, apresentou quadro de baixa acuidade visual detectada há três meses na campanha Veja Bem Brasil realizada na sua cidade em maio de 1998, foi então encaminhado para avaliação no ambulatório de oftalmologia do Hospital das Clínicas da Universidade Estadual de Londrina, associado à baixa acuidade visual havia lesões cutâneas no pé esquerdo pruriginosas e serpiginosas, com história de outros quadros anteriores de larva migrans cutânea tratados. Ao exame oftalmológico apresentava acuidade visual no olho direito (OD) igual a 1,0 e acuidade visual no olho esquerdo igual a conta dedos a 1 metro, os reflexos pupilares estavam normais à direita $\mathrm{e}$ apresentava defeito aferente à esquerda. À biomicroscopia o OD apresentava-se normal e o OE apresentava apenas raras células no vítreo, o exame de fundo de olho revelou-se normal no OD e no OE revelou vitreíte leve, estreitamento arteriolar, atrofia de nervo óptico e lesões esbranquiçadas na retina externa espalhadas pelo fundo de olho próxima às quais foi
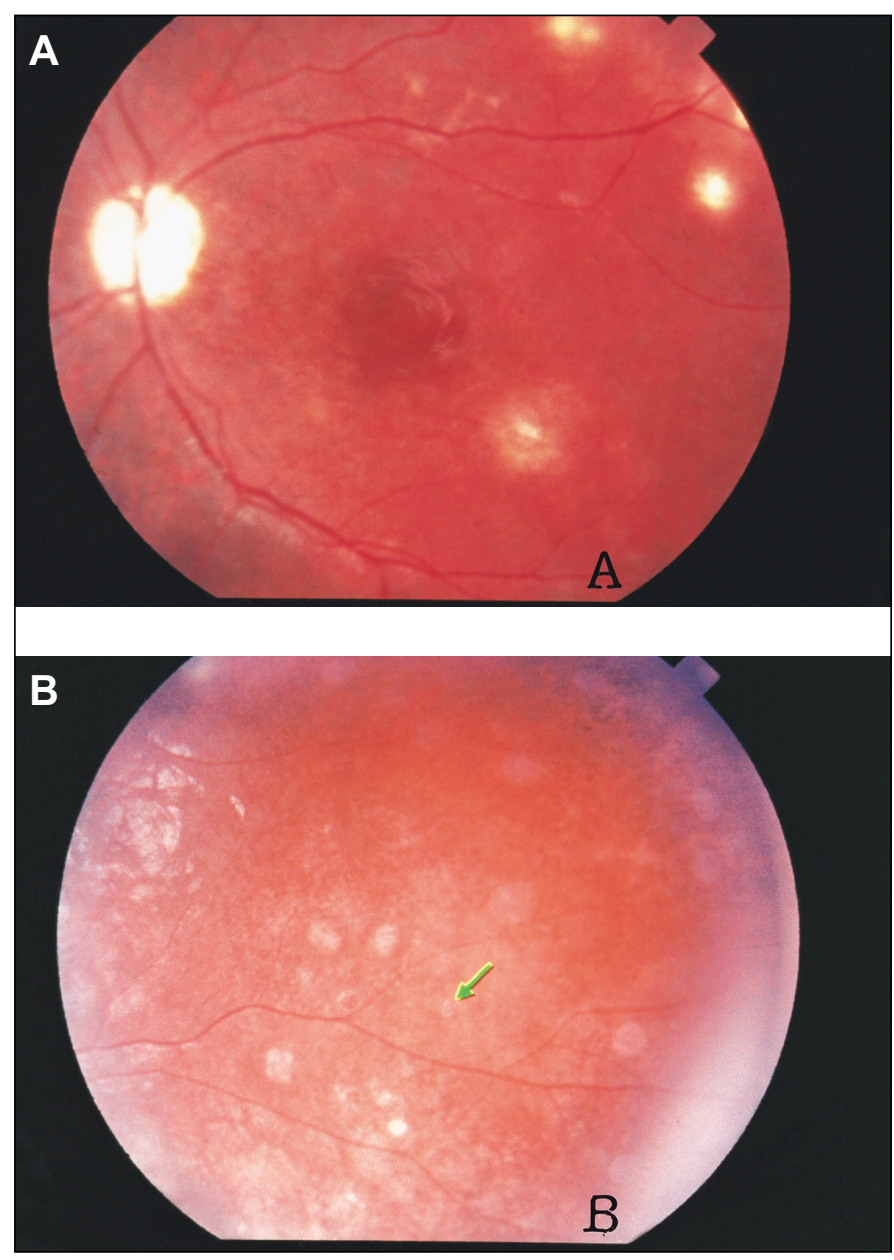

Figura 1 - Retinografia demonstrando: A) Quadro de alterações no epitélio pigmentário, estreitamento vascular e atrofia óptica; B) Larva subretiniana (seta)

evidenciada uma larva móvel (Figura 1). A lesão em pé esquerdo foi avaliada e diagnosticada como larva migrans cutânea (Figura 2). Foram realizados exames subsidiários que mostraram hemograma com discreta eosinofilia, sorologia (ELISA) para toxocaríase negativa e sorologia para toxoplasmose IgM não reagente e IgG 1:4000. A larva subretiniana foi submetida à fotocoagulação a laser de argônio com a destruição da mesma, a lesão no pé esquerdo foi submetida a tratamento com tiabendazol pomada por 10 dias, evoluindo com resolução do processo. O paciente evoluiu com melhora clínica das lesões de fundo de olho (Figura 3) e da acuidade visual para contagem de dedos a 2 metros. As lesões dermatológicas melhoraram com o tratamento clínico.

\section{Análise dos casos}

O estudo dos outros pacientes não mostrou nenhum outro caso com larva migrans cutânea na forma ativa, porém apresentavam quadro de DUSN e sorologia positiva para toxocaríase em três pacientes e antecedentes de larva migrans cutânea em três pacientes, sendo a sorologia e a história de ante- 


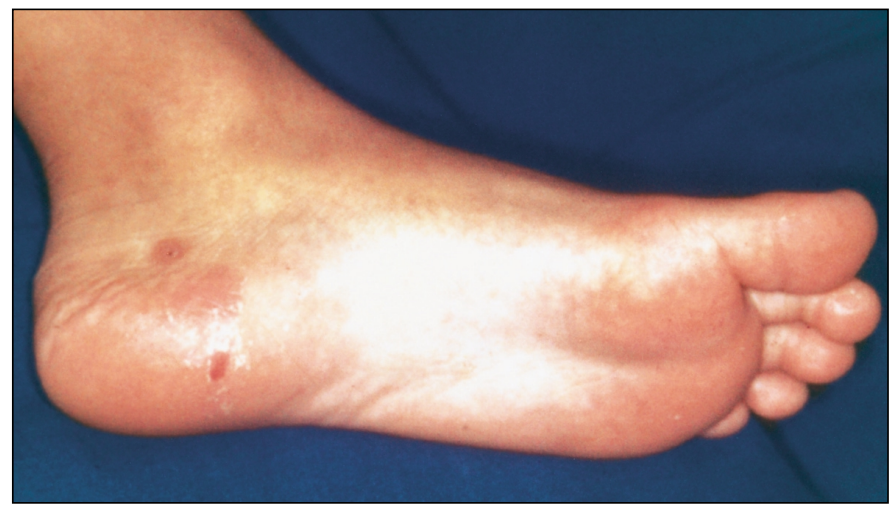

Figura 2 - Lesão cutânia em pé esquerdo (Larva migrans cutânea) em fase ativa - pré-tratamento

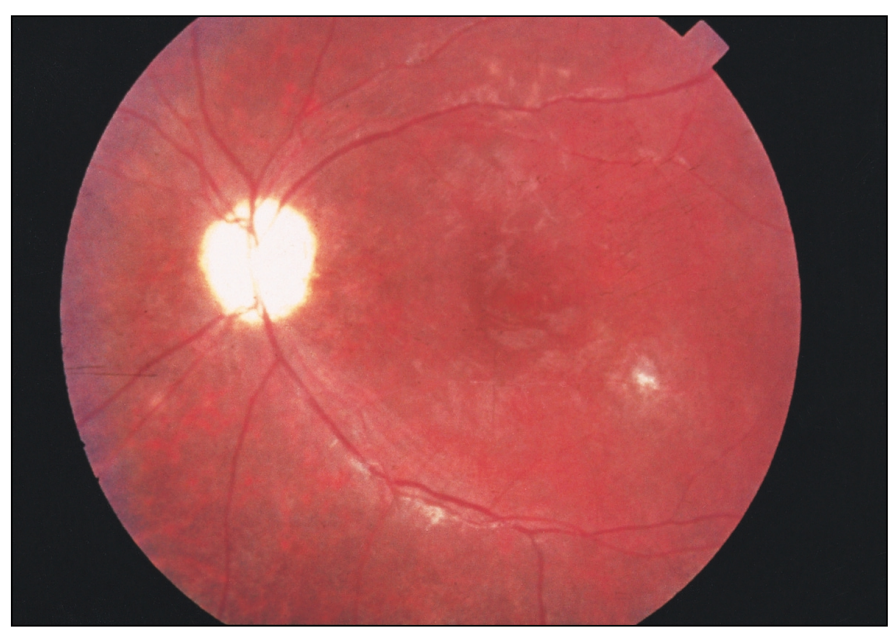

Figura 3 - Retinografia mostrada das alterações do epitélio pigmentário após tratamento (fotocoagulação com laser de argônio)

cedentes de larva migrans cutânea presentes no mesmo paciente em dois casos (números 6 e 7) como mostra a tabela 1.

\section{DISCUSSÃO}

A etiologia da neurorretinite subaguda unilateral difusa continua sendo controversa na literatura tendo sido aventa- das várias hipóteses sobre qual ou quais seriam os agentes causadores, segundo Gass e Braunstein em $1983^{(2)}$ os agentes mais prováveis seriam o Ancylostoma caninum e o Toxocara canis, porém já tendo sido aventados outros nematódios como o Baylisascaris ${ }^{(4,11)}$ que é causador de meningoencefalite e larva migrans visceral em humanos e animais e já foi desenvolvido em modelo animal por Kazakos et al. em 1985(4) produzindo um quadro compatível à D.U.S.N. e encontrado em casos de D.U.S.N. por Goldberg em $1993^{(11)}$, Duke-Elder e Perkins ${ }^{(12)}$ descreveram 4 casos de larvas retinianas com confirmação histopatológica parecendo ser a Onchocerca volvulus em dois casos o Loa loa em um caso e a Wuchereria brancofti no outro caso. No Brasil Souza e Nakashima em $1995^{(8)}$ retiraram a larva de um paciente com D.U.S.N. e de acordo com o exame morfológico existia forte evidência como sendo o Toxocara canis. O Ancylostoma caninum um nematóide de tamanho em torno de 700 micra que geralmente parasita cães é um dos agentes etiológicos da larva migrans cutânea no humano e já foi aventado como causador de D.U.S.N. segundo Gass pelo seu tamanho e características. A história de larva migrans cutânea aparece como antecedente em alguns levantamentos ${ }^{(13)}$, mas não mostrando a associação com lesões ativas e história pregressa de repetidas infestações, este trabalho vem mostrar uma associação entre a larva migrans cutânea ativa e o quadro de D.U.S.N. pela primeira vez na literatura, bem como o levantamento de outros casos com histórico de larva migrans cutânea e sorologia para toxocaríase o que leva à discussão sobre uma etiologia múltipla para este quadro sendo o Ancylostoma caninum um dos seus agentes prováveis, não deixando de esquecer outros agentes causadores de larva migrans cutânea. Seria muito interessante desenvolvermos uma sorologia para o Ancylostoma caninum, e, estamos trabalhado neste sentido.

A B S T RAC T

The autors present a confirmed case of diffuse unilateral subacute neuroretinitis (D.U.S.N.) with identification of the worm in a 6-year-old child with symptoms of cutaneous larva migrans, as well as analysis of 7 other cases of D.U.S.N. and association with cutaneous larva migrans or positive sorolo-

\begin{tabular}{|c|c|c|c|c|c|c|c|}
\hline $\begin{array}{l}\text { 1- Fem. } \\
14 \text { anos }\end{array}$ & $\begin{array}{l}\text { 2- Masc. } \\
20 \text { anos }\end{array}$ & $\begin{array}{l}\text { 3- Masc. } \\
16 \text { anos }\end{array}$ & $\begin{array}{l}\text { 4- Masc. } \\
22 \text { anos }\end{array}$ & $\begin{array}{l}\text { 5- Fem. } \\
16 \text { anos }\end{array}$ & $\begin{array}{l}\text { 6- Masc. } \\
18 \text { anos }\end{array}$ & $\begin{array}{l}\text { 7- Masc. } \\
13 \text { anos }\end{array}$ & $\begin{array}{c}\text { 8- Masc. } \\
6 \text { anos }\end{array}$ \\
\hline$C D$ & CD $1 \mathrm{~m}$ & 0,4 & 0,5 & 0,4 & 0,05 & 0,1 & $\mathrm{CD} 2 \mathrm{~m}$ \\
\hline $\mathrm{Neg}$ & $\mathrm{Neg}$ & $\mathrm{Neg}$ & Pos & $\mathrm{Neg}$ & Pos & Pos & Pos \\
\hline $\mathrm{Neg}$ & $\mathrm{Neg}$ & $\mathrm{Neg}$ & $\mathrm{Neg}$ & $\begin{array}{l}\text { Pos } \\
1 / 64\end{array}$ & $\begin{array}{l}\text { Pos } \\
1 / 32\end{array}$ & $\begin{array}{l}\text { Pos } \\
(1 / 64)\end{array}$ & Neg \\
\hline
\end{tabular}


gy for Toxocara canis. This is the first described case in the literature of the association between D.U.S.N. and cutaneous larva migrans in its active form.

Keywords: Optic neuritis/etiology; Retinitis/etiology; Eye infections, parasitic larva migrans/parasitology; Ancylosto$\mathrm{ma}$ /sorology.

\section{REFERENCIAS}

1. Gass JDM, Gilbert WR Jr, Guerry RK, Scelfo R. Diffuse unilateral subacute neuroretinitis. Ophthalmology 1978;85:521-45.

2. Gass JDM, Braunstein RA. Further observations concerning the diffuse unilateral subacute neuroretinitis syndrome. Arch Ophthalmol 1983;101:1689-97.

3. Casella AMB, Farah ME, Belfort Jr R. Antihelminthic drugs in diffuse unilateral subacute neuroretinitis. Am J Ophthal 1998;125:109-11.

4. Kazacos KR, Raymond LA, Kazacos EA, Vestre WA. The raccoon ascarid. A probable cause of human ocular larval migrans. Ophthalmology 1985;92: 1735-44.
5. Mac Donald HR, Kazaco KR, Schatz H, Johnson RN. Two cases of intraocular infection with Alaria mesocercaria (trematoda). Am J Ophthal 1994;117: 447-55.

6. Oliveira AA, Oréfice F. Estudo de sete casos de neurorretinite difusa subaguda Unilateral. Rev Bras Oftalmol 1991;51:51-5.

7. Souza EC, Cunha SL. Neurorretinite sub-aguda unilateral difusa no Brasil: encontro da larva sub-retiniana. Arq Bras Oftalmol 1992;55:251-4.

8. Souza EC, Nakashima Y. Diffuse unilateral subacute neuroretinitis. Report of transvitreal surgical removal of a subretinal nematode. Ophthalmology 1995; 102:1183-6.

9. Souza EC, Abujanra S, Nakashima Y, Gass DJ. Diffuse Bilateral Subacute Neurorretinitis. First Patient With Documented Nematodes in Both Eyes. Arch Ophtalmol 1999;117:1349-51.

10. Sivberg NB, Jackson RM, Laude TA, Tunnessen WW. Picture of the Month. Arch Fam Med 1998;7:403-6.

11. Goldberg MA, Kazacos KR, Boyce WM, Ai E, Katz B. Diffuse unilateral subacute neurorretinitis. Morphometric, serologic and epidemiologic support for Baylisascaris a causative agent. Ophthalmology 1993;100:1695-701.

12. Duke-Elder S, Perkins ES. Diseases of the uveal tract. In: Duke-Elder S. System of Ophthalmology. St Louis: C V Mosby; 1966. v. 9.

13. Yuen VH, Chang TS, Hooper PL. Diffuse unilateral subacute neuroretinitis syndrome in Canada [letter]. Arch Ophthalmol 1996;114:1279-82.

\section{NOTAS E INFORMAC̣ÕES}

\section{"Como elaborar sua Tese: estrufura e referências"}

Um indispensável roteiro a quem inicia na vida científica (e também a muitos outros que nela estão há já algum tempo), "Como elaborar sua Tese: estrutura e referências" com "modelos de referências para documentos impressos \& eletrônicos", São Paulo, 2001, com 86 páginas (ISBN 85-901897-1-6), tem como autoras Edna Terezinha Rother (Diretora do Centro de Documentação Científica e Coordenadora do Curso de Especialização em Biblioteconomia Médica do I.A.M.S.P.E.) e Maria Elisa Rangel Braga (Diretora da Biblioteca Central da Universidade Federal de São Paulo - Escola Paulista de Medicina). Ambas prestam serviços de Consultoria Técnica aos Arquivos Brasileiros de Oftalmologia desde o ano passado, contribuindo decisivamente no aprimoramento de qualidade de nossas publicações. Objetivo e claro, minucioso e completo, contendo elementos de instrução e variados exemplos sobre como desenvolver formalmente um trabalho acadêmico, o livro é de leitura necessária.

\section{A interessados:}

erother@uol.com.broubragamel@terra.com.br ou, ainda, Livraria Científica Ernesto Reichmann Ltda., Tel. (11) 5575-8283 / 5060-3442 